\title{
eJRIEPS
}

Ejournal de la recherche sur l'intervention en éducation physique et sport

$15 \mid 2008$

Varia

\section{Jeu en déviation et configurations du jeu en football}

\section{Alain Lemoine et Hugues Jullien}

\section{(Q) OpenEdition \\ Journals}

Édition électronique

URL : http://journals.openedition.org/ejrieps/5776

DOI : 10.4000/ejrieps.5776

ISSN : 2105-0821

Éditeur

ELLIADD

\section{Référence électronique}

Alain Lemoine et Hugues Jullien, « Jeu en déviation et configurations du jeu en football », eJRIEPS [En ligne], 15 | 2008, mis en ligne le 01 juillet 2008, consulté le 03 mai 2021. URL : http:// journals.openedition.org/ejrieps/5776 ; DOI : https://doi.org/10.4000/ejrieps.5776

\section{(c) (1)}

La revue eJRIEPS est mise à disposition selon les termes de la Creative Commons Attribution 4.0 International License. 


\section{Jeu en déviation et configurations du jeu en football.}

Alain Lemoine \& Hugues Jullien

Laboratoire de Recherches «EA 3300: APS et Conduites Motrices: Adaptations et Réadaptations », Université de Picardie Jules Verne, Faculté des Sciences du sport, 80025 Amiens, France.

\section{Résumé}

En football, l'accélération de la circulation du ballon ne peut se faire qu'en diminuant le nombre de touches de balle entre les partenaires. Confrontés à des réseaux défensifs très denses les joueurs choisissent souvent de ne plus contrôler du tout le ballon pour dépasser l'adversaire. Ils s'inscrivent alors dans ce que nous appellerons des combinaisons de jeu en déviation qui reposent sur une anticipation collective nécessitant une circulation systématiquement contradictoire de la balle. Les 40 séquences observées montrent une remarquable stabilité spatiale des configurations offensives. Ceci prouve que le jeu en déviation est une option économique, fiable et sécuritaire de progression vers le but adverse. Les attaquants puisent dans la défensive adverse les informations nécessaires à une autoorganisation fulgurante basée sur une simplicité des gestes et de la communication. Nous définirons les principes tactiques nécessaires au bon déroulement de ces combinaisons.

Le geste technique qui permet la transmission du ballon la plus rapide possible est la déviation. Elle consiste à interposer le pied sur la trajectoire du ballon de manière à renvoyer, à volleyer celui-ci vers le coéquipier suivant. A part Dugrand (1989) qui a réalisé un travail approfondi sur la nature et l'apprentissage du jeu en déviation, très peu d'auteurs ont abordé ce thème sous l'angle de l'organisation collective. Ce travail explorera cette composante du jeu en ne retenant que les combinaisons de jeu en déviations (CJED) comportant trois déviations successives avant un tir. A ce niveau de réalisation, le jeu en déviations s'apparente alors à une "activité collective » au sens où l'ont défini Savoyant \& Bouthier (1985) et non au simple hasard. Si aujourd'hui le football se pratique dans un contexte de 
pression temporelle important, le jeu en déviations apparaît comme une circulation du ballon intéressante pour tenter d'échapper aux défenses adverses. (FIFA, 1986).

Les difficultés méthodologiques inhérentes à l'observation des actions collectives en football empêchent souvent des mesures précises pénalisant d'autant l'analyse théorique. Certains travaux reposent alors sur des situations décontextualisées dans lesquelles les joueurs doivent résoudre des tâches de un contre un (Lerda, 1993), ou bien encore tirer un penalty (Henneman, 1989; Mc Morris \& al, 1993). A l'inverse, l'utilisation comme support expérimental d'un jeu réduit à 5 contre 5 , permettra l'étude d'actions collectives en situation écologique. Dans ces conditions d'opposition réelle, la quantité d'information produite par le mouvement du ballon et celle produite par les mouvements des joueurs nous apparaît être un indicateur nouveau pour caractériser les actions collectives entreprises par les attaquants.

Après avoir défini le cadre théorique, nous décrirons la construction de notre outil de mesure et les variables qui rendent compte des déplacements des joueurs et du ballon. Nous montrerons ensuite comment les attaquants organisent leurs déplacements et la circulation du ballon afin de créer et maintenir une circulation de balle ininterrompue en jeu dévié afin de produire chez les défenseurs un désordre organisationnel fatal ayant pour conséquence une incapacité momentanée à s'organiser collectivement pour défendre la cible.

\section{Cadre théorique}

Parce qu'il repose sur des interactions d'éléments appartenant à deux équipes qui s'opposent à l'intérieur d'un espace délimité, le football peut être considéré comme un système complexe et dynamique (Gréhaigne, 1992; Gréhaigne \& Godbout, 1995; Braida, 2003, Mouchet, 2005). Pour comprendre son fonctionnement, l'analyse systémique fournira deux axes fondamentaux (Walliser, 1977) :

- la notion de sous-systèmes en interdépendance assurant à l'ensemble sa cohérence

- la notion de modification dans le temps et la permanence de l'ensemble

Replacé dans ce contexte systémique l'action collective des attaquants qui dévient le ballon entre eux dans le but de dépasser la défense et faire basculer le rapport de forces met en évidence une régulation adaptative collective du sous-système attaque en réponse à une organisation momentanée du sous-système défense (Gréhaigne, 1990). La déviation apparaît comme une réponse tactique collective au problème posé par le jeu (Malhö, 1974, Menaut, 1998). 


\section{1. Le jeu réduit}

Le cadre expérimental choisi propose un jeu réduit qui « permet la mise en place d'un rapport de force qui oriente le jeu vers une entrée rapide en zone de marque, et valorise le jeu en anticipation » (Deleplace, 1979). Plus généralement, cette situation, bien que réduisant le nombre des éléments, propose « une intégration de tous les paramètres du jeu » (Gréhaigne \& al, 1999) et respecte la réversibilité des actions. Le jeu réduit reproduit certains aspects du jeu à 11 et favorise à priori « la circulation rapide du ballon » par « un réseau d'échange en losange » (Mombaerts, 1999). Même si la situation minore à priori d'autres paramètres comme le jeu long ou la progression du ballon en relais par le milieu de terrain, il n'en demeure pas moins que la situation respecte la complexité du jeu réel et met en évidence des formes de relations entre 3 ou 4 joueurs « base des mouvements tactiques du football à $11 »$ (Dugrand, 1989).

\section{2. L'Information comme témoin des actions collectives}

Pour assurer la circulation ininterrompue du ballon, les attaquants se déplacent. Ces mouvements génèrent 3 types d'Information mesurables au moment de la déviation du ballon et nécessaires à chacun des joueurs pour assurer la poursuite de l'action collective. La classification décrite par Famose (1983) sera reprise de la façon suivante :

- $\quad$ L'Information Spatiale (I.S.) : où, dans quelle direction est déviée la balle ?

- $\quad$ L'Information Evénementielle (I.E.) : à qui parmi les partenaires est déviée la balle ?

- $\quad$ L'Information Temporelle (I.T.) : à quel moment est déviée la balle ?

La valeur de l'Information (I), sera donnée par la formule $\mathbf{I}=\log _{2} \mathbf{1} / \mathbf{P}$ où $P$ représente la probabilité d'apparition d'un événement (Shannon \& Weaver, 1945 ; Bakker, 1992). Issue aussi des théories de l'information, la redondance $(R)$ d'un système traduit le fait « qu'un signal annonce sans ambiguïté l'apparition des suivants » (Chappuis, 1967). Elle rend compte de la qualité des relations entre les joueurs qui cherchent à limiter l'incertitude entre eux (Moles, 2002). Sa valeur sera calculée à partir de la formule $\mathbf{R}=\mathbf{1 - H / H m a x}$ (bits) où $H$ est la quantité totale d'information produite par les attaquants et Hmax la quantité d'Information en absence de redondance. Elle varie de 0 à 1 quand l'incertitude entre les partenaires est nulle. 


\section{Méthodes}

L'espace expérimental était un terrain mesurant $50 \mathrm{~m}$ de long sur $25 \mathrm{~m}$ de large. II permettait une prise de vue en plan unique et fixe. Une caméra analogique Sony type CCD munie d'un grand angle était placée sur une nacelle à $20 \mathrm{~m}$ du sol derrière un but dans l'axe longitudinal du terrain. Les bandes ont été exploitées sur un magnétoscope Sony SLV E-930 permettant la lecture image par image. La durée des rencontres était de $4 \mathrm{~min}$ permettant aux joueurs d'être au maximum de leurs ressources à chaque passage. Les 8 équipes étaient constituées de 5 joueurs de niveau régional capables de jouer en déviations. Les rentrées de touche étaient réalisées au pied et les corners à la main. La règle du hors-jeu n'était pas appliquée. Le protocole stipulait qu'une équipe jouait librement alors que l'autre jouait en une touche de balle pendant toute la durée de la rencontre. L'observation systématique des séquences vidéo (W. Dufour, 1989; Hugues \& Francks, 1997) a permis d'obtenir 40 combinaisons respectant le critère des 3 déviations réussies avant le tir.

2. 1. La mesure de l'Information

Pour mesurer l'Information Spatiale, nous avons identifié 8 déviations possibles en fonction de la direction de la passe (Fig. 1).

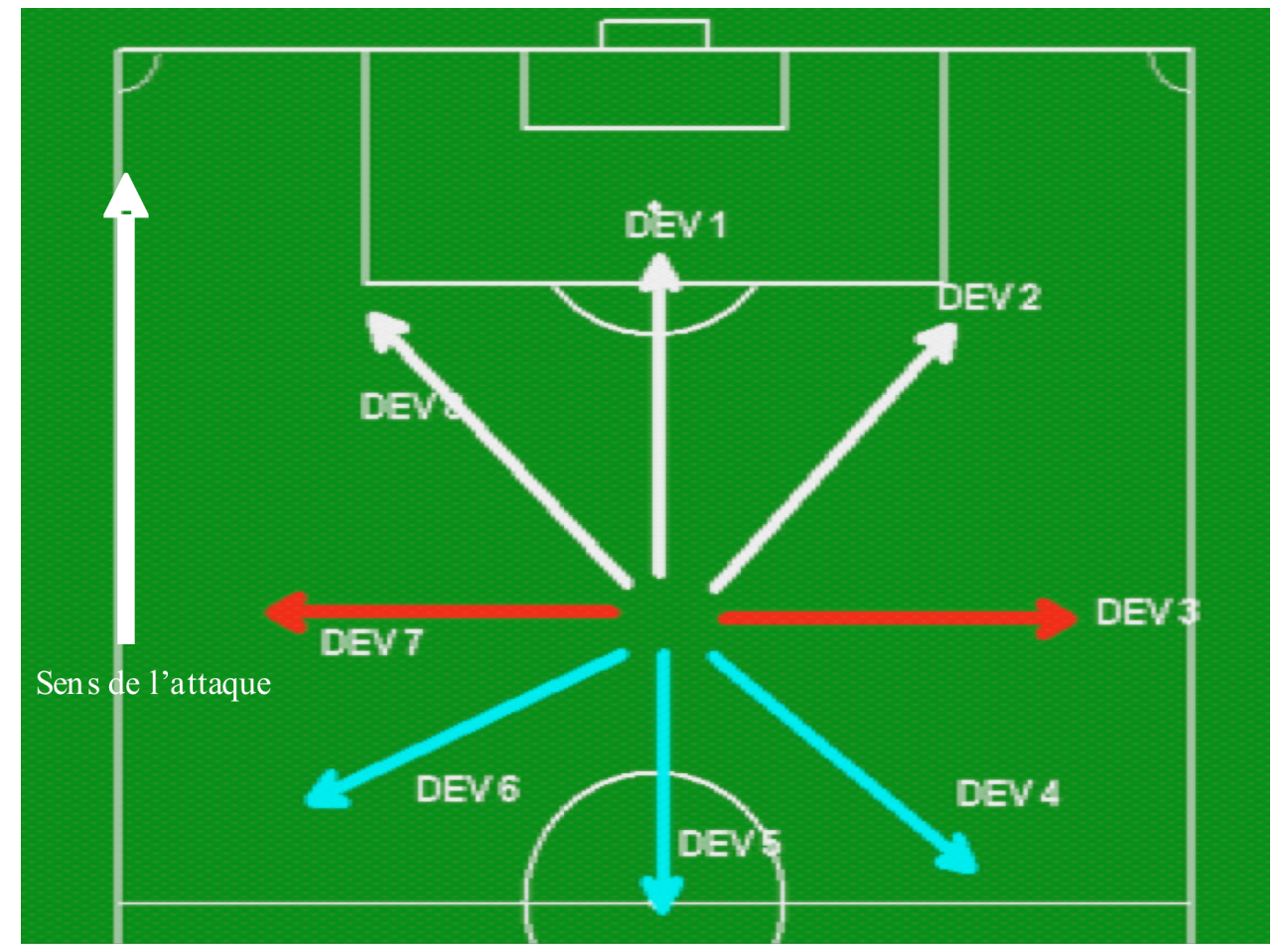

Figure 1. Les différentes déviations possibles en fonction de l'espace. Les déviations ainsi identifiées explorent 3 directions et sont caractérisées par l'angle qu'elles forment avec l'axe but-but égal à $0^{\circ}$. 
Le changement de direction du ballon entre deux déviations successives était lui aussi observé.

La mesure de l'Information Evénementielle a été faite en déterminant 3 possibilités de passes pour le Porteur de Balle. La première représentait une seule et unique possibilité de déviation, la seconde était le cas où la balle pouvait être déviée vers plusieurs partenaires. Enfin, la troisième était la situation où il semblait impossible à l'observateur que le ballon puisse être dévié. L'Information Temporelle était définie par le temps mis par le joueur pour dévier la balle. 4 cas ont été identifiés : celui de la déviation immédiate ; celui des déviations effectuées après la pose d'un appui au sol ; celui des déviations faites après un changement d'orientation des appuis au sol (1/2 tour, $1 / 4$ de tour); enfin celui des déviations faites après une feinte. La surface de contact utilisée (intérieur ou extérieur du pied) pour dévier la balle n'a pas été étudiée. Cet aspect technique du jeu en déviation mérite une étude spécifique qui reste à faire.

\section{2. La validité de l'outil de mesure}

Pour respecter les protocoles scientifiques en usage dans les processus d'élaboration des outils de mesure, 12 experts ont été sollicités (Reboul-Marty \& Launois, 1995, ANAES, 2003). Ils ont visionné une séquence test et leurs résultats ont été soumis au test non paramétrique de Kappa qui évalue "la concordance réelle entre différents juges " (Le Bozec, 2002) et chiffre l'intensité de l'accord réel entre les observateurs. K est donné par la formule : K= $P a$ $P e / 1-P e$. L'accord entre les juges est d'autant plus élevé que $\mathrm{K}$ est proche de 1 . Les valeurs trouvées sont bonnes, $\mathrm{K}=0.751$ pour I.S., 0.653 pour I.E et 0.620 pour I.T.

\section{3. Les relevés topographiques}

Pour compléter la mesure de l'information et comprendre la dimension spatiale des mouvements des joueurs, il a été fait des relevés topographiques des combinaisons de jeu au moment de chaque déviation. (Gréhaigne, 1990). A partir de l'image, la position des attaquants et des défenseurs était relevée, puis un logiciel spécifique construisait un schéma où l'axe principal de l'attaque croisait son axe de dispersion au niveau du centre de gravité caractérisant ainsi la position des attaquants dans l'espace. La position relative des axes offensifs et défensifs l'un par rapport à l'autre permettait aussi de montrer à chaque instant la situation du rapport de forces entre les 2 équipes. Trois statuts de la défense ont été établis par rapport à l'attaque : défense en barrage, en équilibre ou en poursuite (Tab. II). 
Tableau II. Typologie des combinaisons Attaque/Défense.

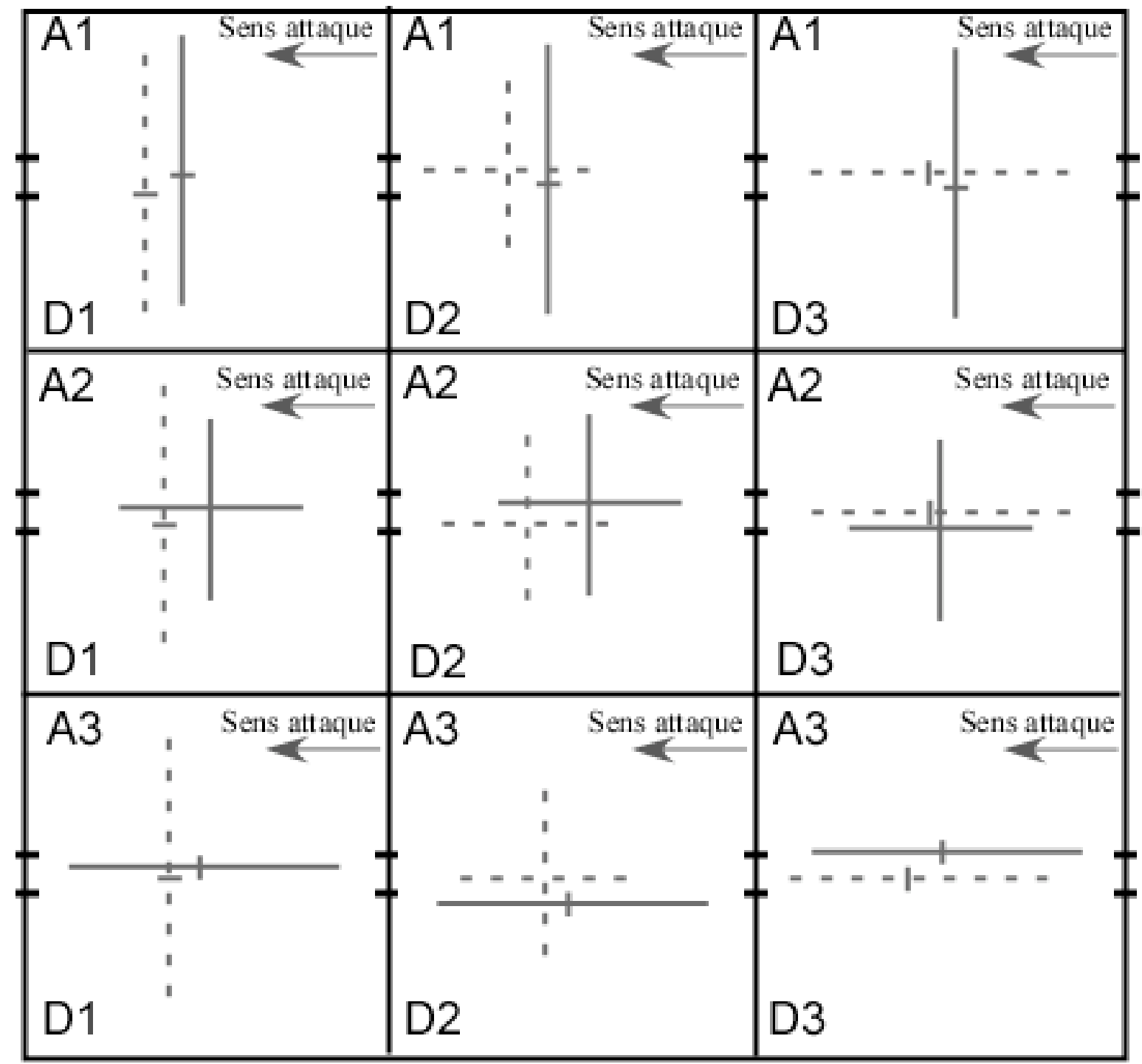

Légende : Trait plein, axe principal et axe de dispersion de l'attaque, pointillés, ceux de la défense.

A1: correspond à une position des attaquants écartés les uns des autres et peu étalés sur la longueur du terrain.

D1 caractérise une défense plutôt à plat qui est écartée et compacte. Elle offre peu d'espaces libres entre ses éléments.

A2 est une configuration où les attaquants sont globalement disposés sur 2 lignes et leur placement permet un passage du ballon de l'arrière vers l'avant.

D2 correspond à une défense généralement étagée en 2 lignes qui présente des espaces entre ces éléments. Les parties latérales du terrain sont vulnérables.

A3 est une configuration d'attaque dont l'axe principal est étendu dans la profondeur du terrain et l'axe de dispersion très réduit. Les attaquants sont répartis et étalés dans la profondeur du terrain. Ils sont peu écartés les uns par rapport aux autres.

D3 présente des défenseurs étalés sur la longueur du terrain et écartés les uns des autres. Cette situation est le signe d'une certaine vulnérabilité de la défense. 


\section{4. Statistiques}

Les valeurs utilisées dans l'article représentent la moyenne des différentes mesures aux 3 temps de l'attaque \pm l'écart type. La comparaison entre les différentes moyennes a été réalisée à partir d'un test paramétrique pour des échantillons appariés ( $t$ de Student). Le seuil significatif a été fixé à $p<0.05$.

\section{Résultats}

\section{1. L'information Spatiale}

L'évolution de la valeur de l'Information Spatiale produite au cours des Combinaisons de JED est faible. Sa valeur totale moyenne est de $2.655 \pm 0.22$ bits et le test $t$ de Student montre que les écarts entre ses différentes valeurs tout au long de l'attaque ne sont pas significatifs. La fréquence d'utilisation par les attaquants des différents types de déviations montre que Dev1 varie peu et vaut pour $12.50 \%$ en moyenne des déviations utilisées. Les Dev2 et Dev8 présentent, elles, des évolutions parallèles remarquables dans la mesure où quand l'une diminue l'autre augmente. On assiste alors à une alternance des " préférences » de l'espace exploré. Au début de l'attaque les attaquants dévient plutôt sur la gauche du terrain Dev8 et Dev7 représentent $37.50 \%$ et sont majoritaires alors qu'au moment de la seconde déviation les attaquants dévient plutôt vers la droite avec Dev2 et Dev3 qui valent pour $40.00 \%$ des cas. Dev8 est la déviation la plus utilisée par les joueurs avec $33.33 \%$ des cas en moyenne.

En ce qui concerne les changements de direction dans $95.83 \%$ des cas le ballon est dévié dans une direction différente de la précédente.

\section{2. L'information événementielle}

Les chiffres de l'information événementielle montre une grande stabilité dans le temps : 1.316 bits \pm 0.13 de moyenne. Le $t$ de Student montre aussi que les différences d'un moment à l'autre ne sont pas significatives. II y a peu de courses de démarquage et les appels de balle ne sont présents que dans $50 \%$ des attaques. Quand ils existent, les appels ne sont le fait que d'un seul joueur dans $78,7 \%$ des cas. Les courses sont majoritairement orientées vers l'avant et les attaquants changent très peu de direction. Ce qui est l'inverse pour les défenseurs. Les résultats montrent aussi que les attaquants sont majoritairement en mouvement à vitesse lente et que les appels de balle ne surviennent le plus souvent qu'au moment de la déviation qui précède le tir. 
3. 3. L'Information Temporelle

La valeur moyenne de I.T. est de 1.232 bits \pm 0.27 . Le $t$ de Student montre que pendant l'attaque il n'y a pas de différence significative entre les comportements des attaquants. II n'y a que $5 \%$ des cas où les joueurs produisent des feintes. A l'inverse dans $77.50 \%$ des cas, les attaquants dévient le ballon sans temps d'attente, sans volonté de masquer le moment de la déviation.

La valeur de la Redondance oscille entre $0.665 \pm 0.02$ et $0.634 \pm 0.04$. Cela montre à la fois une stabilité de $\mathrm{R}$ tout au long de la CJED et d'autre part la qualité de l'information produite entre les attaquants ( $R$ ayant sa valeur maximum à 1$)$. La faible valeur des écart-types montre l'homogénéité des résultats.

\section{4. Evolution synchronique et diachronique}

Au début de la combinaison, les attaquants s'organisent majoritairement sur la largeur du terrain en A1 (62.50\%) et A2 (12.50\%), leur dispersion est faible. Au moment de la seconde déviation, l'occupation de la largeur du terrain s'accentue au détriment des attaques allongées (A3). A l'instant de la dernière déviation avant le tir, l'attaque est toujours majoritairement organisée à partir d'une structure privilégiant l'occupation de la largeur du terrain et un écartement faible entre les joueurs.

En défense, il y a une tendance forte à l'augmentation progressive des organisations de type D3 pour atteindre un pourcentage très important (82.50\%) au moment du tir. L'évolution diachronique des CJED a été mesurée par le nombre de changements de structures identifiés dans le temps. 5 cas sont apparus. Le premier regroupe des CJED qui ne subissent aucune modification (2,50\%). Le deuxième est constitué des CJED qui voit 1 changement se produire $(40 \%)$. Le troisième groupe est constitué des CJED qui se modifient très peu avec des structures voisines (25\%). Les quatrième et cinquième groupes sont ceux qui voient 2 $(17,50 \%)$ et 3 modifications au cours des CJED (15\%).

Il y a peu de CJED qui voient leurs structures se modifier profondément au cours de l'attaque. $40.00 \%$ des cas répertoriés sont des CJED où le rapport de forces n'est modifié qu'après 3 premiers temps de stabilité dans une même disposition. Exemple: A1/D1-A1/D1-A1/D1A1/D3. $25.00 \%$ des cas sont des CJED où la modification d'une structure à l'autre n'est pas un changement radical du rapport de forces, mais plutôt une évolution limitée de l'Espace de Jeu Effectif (EJE) produit par les attaquants. Si on ajoute le cas des CJED sans aucun 
changement on peut constater que le JED se développe majoritairement (67.50\%) sans modifier de façon importante l'EJE défini dès la $1^{\text {ère }}$ déviation.

Au début de l'attaque la défense dans $85.00 \%$ des cas est en barrage face à l'attaque. C'est encore vrai pour T2 et $\mathrm{T} 1$ avec respectivement $75.00 \%$ et $52.50 \%$ des défenses recensées. Pendant le tir les défenseurs sont en poursuite dans $80.00 \%$ des cas, ce qui coïncide avec la forme fréquemment relevée de structure D3. A ce moment, les défenseurs sont étirés sur la longueur du terrain (axe principal) et même si leur axe de dispersion est faible le rapport de forces devient favorable aux attaquants.

\section{Discussion.}

A ce stade de nos investigations, le jeu en déviations en situation de jeu réduit s'apparente à un système auto-organisé qui puise dans la complexité du jeu les conditions nécessaires à son fonctionnement (Mc Garry \& Al, 2002). II possède «la capacité d'utiliser les phénomènes aléatoires pour les intégrer, et les faire fonctionner comme des facteurs créateurs d'ordre » (Atlan, 2002). En effet, les différents résultats montrent que les attaquants construisent un espace de jeu effectif stable garant d'une communication simplifiée entre eux permettant alors une circulation du ballon instantanée et ininterrompue face à des défenseurs qui restent actifs tout au long des actions étudiées.

La faible valeur de la redondance et sa stabilité dans le temps, traduisent la stabilité de l'Espace de Jeu Effectif tout au long de l'attaque. L'amplitude des déplacements offensifs est peu importante et les joueurs ont des mouvements peu variés et peu saturés en Information. Les attaquants créent les conditions d'une redondance initiale en utilisant majoritairement des attaques de type $\mathrm{A} 1$ et $\mathrm{A} 2$ au moment de la première déviation. Ces dispositifs sont maintenus ensuite tout au long des combinaisons ce qui assure une stabilité diachronique au système JED. Ainsi disposés, les attaquants "proposent une auréole de possibilités » (Dufour. J, 1974) soit en soutien ou en appui du PB. Le fait que dans $67.50 \%$ des cas il n' y a pas de changement du type d'attaque entre 2 configurations de jeu intermédiaires montre bien que les attaquants maintiennent ces structures spatiales sécuritaires pour la circulation du ballon jusqu'au moment du tir assurant ainsi une redondance évolutive à l'attaque. C'est grâce à cette stabilité spatiale et informationnelle que le JED, confronté aux actions défensives, s'auto organise et produit une circulation de balle déviée permettant à terme le dépassement des défenseurs. La création d'ordre au niveau offensif passe par la production 
de certitude pour les partenaires (stabilité des positions (I.E faible), stabilité du type de transmission (I.T faible) et d'incertitude vis-à-vis des adversaires (où va aller la balle ?). La déviation devient le paramètre fondamental d'une information univoque entre les attaquants, elle même rendue possible par les déplacements offensifs coordonnés des attaquants. Le modèle du JED proposé renvoie une image des 4 attaquants se déplaçant de manière simultanée en préservant la cohérence spatiale de l'ensemble (Fig. 2).

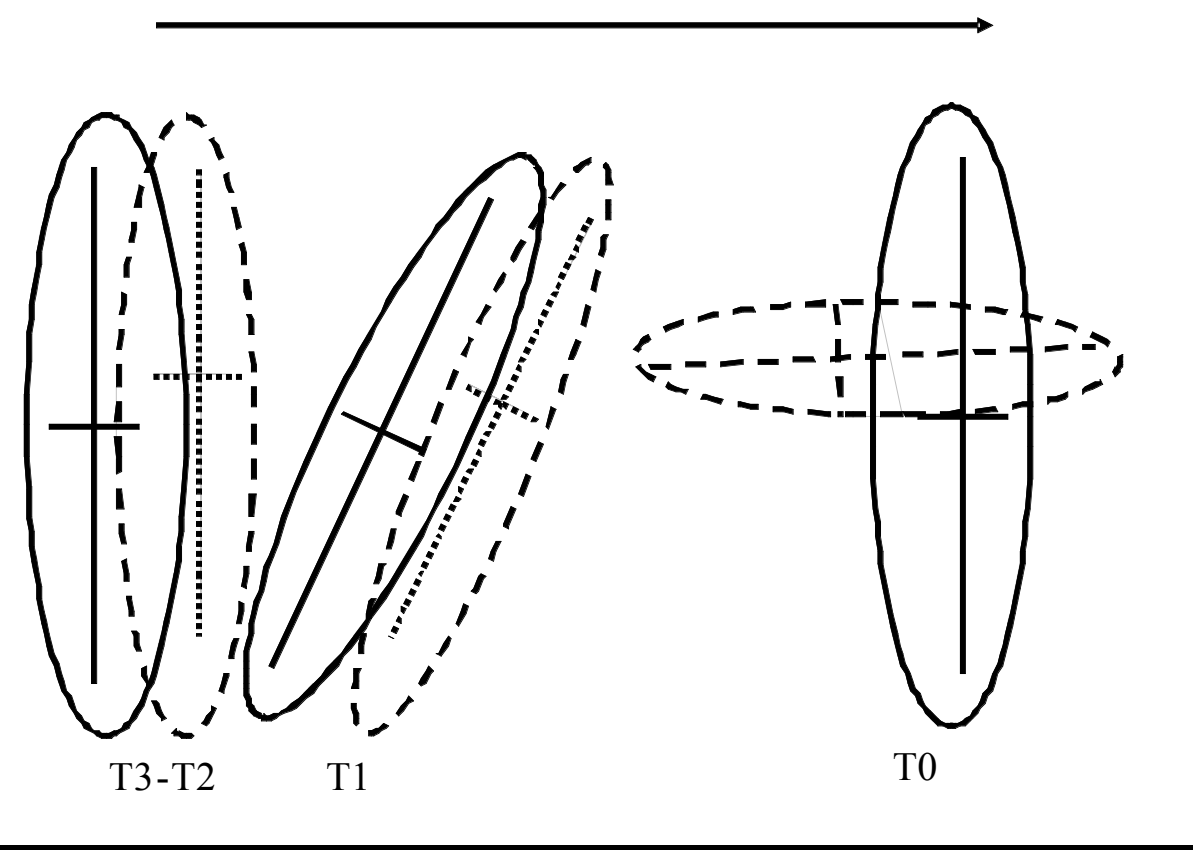

Figure 2. Modèle du JED.

Légende : En trait plein le sous-système attaque et en trait pointillé et grisé le sous-système défense.

Dans la figure 2, le cercle englobant les axes de l'attaque schématise l'espace de circulation du ballon qui est « pré » établi dès T3. L'EJE offensif varie peu et la circulation du ballon est circonscrite dans cette surface. L'axe principal de l'attaque est de type A1 et il se maintient tout au long de la CJED. A l'inverse, le sous-système défense qui est en barrage en T3 voit son axe principal se modifier dans le temps et passer progressivement de D1 à D3 en T0. A ce moment, les défenseurs sont en poursuite et ne peuvent plus intervenir efficacement.

Le JED se caractérise par un espace de transmission du ballon anticipé, optimisé et stabilisé. Le non porteur de balle anticipe son placement pour permettre la continuité de la CJED et dévier la balle instantanément. Cette action individuelle n'est possible qu'en cohérence avec celles des autres partenaires. La quantité et la clarté des informations liées aux flux du 
système sont alors suffisantes pour que les attaquants restent organisés et mettent les défenseurs en difficulté.

4. 1. Le JED, une attaque en 2 temps

Pour aller à leur terme les CJED ont un cheminement du ballon en 2 temps. Le premier est régulier jusqu'à la $3^{\text {ème }}$ déviation ce que confirme la valeur de la redondance et la stabilité de I'EJE. II permet une conservation sécuritaire du ballon, dans un rapport de forces qui reste le plus souvent à l'avantage de la défense avec $85.00 \%$ des défenses en barrage.

Le second temps, très bref au moment de la dernière passe, marque un changement de rythme du ballon net, une accélération permettant à l'attaque de dépasser la défense de manière définitive et coïncide avec l'utilisation maximale de Dev1. La bascule du rapport de forces entre l'attaque et la défense s'engage. A cet instant, les attaquants n'ont plus intérêt à jouer latéralement, au contraire pour mettre le partenaire en position de tir, il est pertinent de jouer vers l'avant. L'utilisation de la déviation, comme dernière passe avant le tir, supprime les possibilités de réorganisations défensives. Entre $\mathrm{T} 1$ et $\mathrm{T} 0$, ce sont $82.50 \%$ des défenses qui sont en D3 dont $30.00 \%$ qui basculent à ce moment là sous l'action offensive. Le soussystème défense qui jusqu'à là s'appuyait principalement sur des structures étalées sur la largeur, voit ses axes basculer et s'étirer sur la longueur du terrain tout en se retrouvant en poursuite. La non application de la règle du hors-jeu favorise évidemment les déviations faites dans la profondeur du terrain, et est une limite à prendre en compte pour expliquer ces différents phénomènes.

Sans avoir mesuré I.S au moment du tir, nous constatons que la défense est le plus souvent dépassée. II y a donc bien une rupture nette dans la nature de la relation attaque-défense qui s'opère ici. Elle correspond au fait que les défenseurs ne peuvent plus combler le déficit spatio-temporel imposé par l'attaque, ce qui se traduit par un retard vis à vis du tireur quand il se retrouve en un contre un face au gardien de but (Lemoine, 1996).

4. 2. Une circulation de balle particulière

Le fait que la répartition des espaces explorés tout au long des 40 CJED soit quasi stable nous incite à penser que les attaquants dévient le ballon souvent de la même façon, voire avec les mêmes "routines ». C'est la succession ininterrompue des différentes directions contradictoires imposées au ballon qui met la défense en difficulté en créant de l'incertitude.

D'un point de vue technique la progression du ballon s'appuie sur une systématisation de la passe faite dans une direction opposée à celle de la déviation précédente. Le jeu de 
« zigzags » devient un élément structurant du JED car il organise le cheminement de la balle jusqu'au moment du tir. A l'inverse, le jeu direct dans l'axe profond du terrain ne représente que la $3^{\text {ème }}$ solution utilisée par les attaquants. Si l'on se réfère à l'analyse de Wrzos (1984), on s'aperçoit que le JED utilise deux fois moins les passes dans l'axe du but que le Jeu en «touches multiples ». Cette caractéristique montre que le JED privilégie le jeu « à l'oblique » par rapport au jeu direct.

Pour ce qui est du traitement de l'Information, quand les défenseurs sont confrontés à une circulation ininterrompue du ballon, ils sont obligés de prendre, sans arrêt, des indices sur la destination de la balle pour organiser leurs propres actions. Quand le PB ne contrôle pas le ballon et qu'il le dévie dans une direction opposée ou oblique à celle où l'arrière vient de s'engager, il devient malaisé pour les défenseurs de réorganiser un déplacement individuel et collectif efficace face à l'attaque.

Ici nos résultats confirment une "idée " perçue par les techniciens quand ils disent qu'il faut jouer à contre pied de l'adversaire. Ces différentes notions expliquent aussi la mise hors de position de la défense juste avant le tir. En effet, celle-ci survient à la fin de l'attaque et la succession des 3 déviations avant le tir produit une sorte de "cumul d'Incertitude spatiale » pour le défenseur qui, au fur et à mesure de l'attaque voit la distance qui le sépare des attaquants augmenter, pour finalement provoquer une incapacité " finale » et irréversible à s'opposer au tir. Nous confirmons ici encore des connaissances « de terrain » exprimées par Mombaerts (1991) quand il avance que lorsque « le ballon circule vite, systématiquement, par des passes redoublées d'un joueur à l'autre, les défenseurs semblent comme privés d'initiative, les actions s'enchaînent avec une telle rapidité que leur lisibilité devient impossible ».

L'utilisation de la notion de redondance du système montre que les attaquants s'organisent pour changer à minima les structures spatiales de la CJED. Les déplacements modifient peu l'Espace de Jeu Effectif et ils sont principalement orientés vers la cible. Ce qui compte davantage que la vitesse ou la direction de la course des attaquants, c'est le moment où l'appel de balle final est déclenché (le plus souvent au moment de la dernière déviation avant le tir). Le jeu en déviations apparaît alors comme un jeu économique où le mouvement continu est dominant, où tout est mis en œuvre pour qu'au milieu d'un environnement stable crée et entretenu par les attaquants, le ballon circule de façon systématiquement contradictoire, « rebondissant » d'un joueur à l'autre pour devenir insaisissable pour les 
défenseurs. L'utilisation de la déviation permet une simplification des informations échangées entre partenaires. Ce gain de temps est lui même amplifié par l'instantanéité de la passe qui augmente d'autant l'ascendant pris par les attaquants sur les défenseurs. Le choix fait par les joueurs de jouer en déviation dans une configuration momentanée, suppose alors une adhésion à "un authentique projet collectif de jeu » concept que nous pourrions rapprocher de la notion "d'action collective située" (Salembier \& Pavard, 2004) et la création d'un référentiel commun de décision.

\section{Conclusion}

Ce travail a permis de confirmer certaines connaissances de terrain déjà énoncées par les techniciens: jouer à contre-pied de la défense, rechercher la passe en profondeur pour mettre la défense en difficulté. A l'inverse, elle a aussi montré que le jeu en déviations ne demande pas aux joueurs de se déplacer vite et dans toutes les directions du terrain. Au contraire conserver et progresser sans contrôler la balle revient à organiser un espace de circulation du ballon stable tout au long de l'attaque, ce qui demande aux joueurs de s'auto organiser dans le cadre d'une action collective située dans le temps et l'espace.

Dans l'état actuel de ce travail, les conclusions s'appliquent au jeu réduit à 5 contre 5 et ne peuvent être transposées sans précaution au jeu à 11. Le passage rapide de la zone défensive à la zone offensive, peut avoir limité les phases-mère du jeu, c'est à dire " le lieu d'apparition des déséquilibres à l'origine des attaques" (Gréhaigne et al, 2005). De prochaines investigations centrées sur le jeu à 11 devraient permettre d'approfondir ces premiers résultats en mettant en évidence les facteurs de déclenchement du jeu en déviations dans les conditions réelles du match.

\section{Bibliographie}

Agence Nationale d'Accréditation et d'Evaluation en santé. (2003). Validation des échelles d'évaluation. Lu le 10 février 2005 sur http.//.anaes.frl:ANAES/publications.nsf.

Atlan, H. (2002). Auto-organisation [CD-ROM]. Encyclopédie Universalis.

Bakker, F.C., Whitting, H.T.A, \& Van der Brug, H. (1992). Psychologie et pratiques sportives. Paris : Vigot. 
Bonnardel, P. (2003). Le coefficient de Kappa. Lu le 10 février 2005 sur http:// kappa.chez tiscali.fr/kappa_intro.htm.

Braida, J.P. (2003). A propos de football : entre complexité et créativité. Just'au corps, 2, 203227.

Chappuis, R. (1967). Introduction à l'étude de la perception en situation de jeu collectif. Revue EPS, 87. 31-35.

Deleplace, R. (1979). Rugby de Mouvement - Rugby total. Paris : Edition Revue EPS.

Dufour, J. (1974). Football moderne. Paris : Bornemann.

Dufour, W. (1989). Les techniques d'observation du comportement moteur. Revue EPS, 217 , 68-73.

Dugrand, M. (1989). Football, de la transparence à la complexité. Paris: PUF .

F.I.F.A. (1986). Rapport officiel de la Coupe du Monde 1986.

Famose, J.P. (1983). Stratégies pédagogiques, tâches motrices et traitement de l'information. In J.P. Famose, J. Bertsch, E. Champion, \& M. Durand (Eds.), Tâches motrices et stratégies pédagogiques en éducation physique et sportive (pp. 9-21). Paris: Editions EPS.

Grehaigne, J.F. (1990). Analyse des mouvements collectifs précédant un but en football. Science et Motricité, 12, 41-53.

Grehaigne, J.F. (1992). L'organisation du jeu en football. Paris: Actio.

Gréhaigne, J.F., Godbout, P. (1995). Tactical knowledge in team sports from a constructivist and cognitivist perspective. Quest, 45, 490-505.

Gréhaigne, J.F., Godbout, P., \& Bouthier, D. (1999). The foundation of tactics and strategy in team sports. Journal of Teaching in Physical Education, 18, 159-174.

Grehaigne, J.F., Caty, D., Billard, M., \& Chateau, L. (2005). Les concepts de matrice offensive et défensive envisagés sous l'angle de la didactique des sports collectifs. eJRIEPS, 7, 20-41.

Henneman, M.C. (1989). Les ajustements préparatoires. In A. Vom Hoffe (Ed), Taches, traitement de l'information et comportement dans les APS (pp. 161-191). Issy les Moulineaux : Ed EAP.

Herbin, R., \& Rhetacker, J.P. (1976). Football. Paris: R.Laffont.

Hugues, M., \& Francks, I. (1997). Notational analysis of sport. London: E \& FN Spon. 
Landis, J.R., \& Koch, G.G. (1977). The Measurement of Observer Agreement for Categorical Data. Biometrics, 7a, 33, 159-174.

Laurier, A. (1993). Football, culture tactique et principes de jeu (3ème ed). Paris: Chiron.

Le Bozec, C. (2002). Tests diagnostiques, DCEM1 d'informatique médicale et technologies de la communication. http://www.cybermed.jussieu.fr/Broussais/InforMed/ Enseignement/DCEM1.

Lemoine, A. (1996). Observation et analyse du jeu en passes rapides utilisé par une ligne offensive pour dépasser un bloc défensif. Mise en évidence des moyens utilisés ainsi que des différents processus sollicités chez le joueur. Mémoire non publié de DEA STAPS, Université de Paris X Nanterre.

Lerda, R. (1993). Les déterminants cognitifs des conduites de décision en football. Thèse de doctorat en STAPS, Université de Aix-Marseille II.

Mahlo, F. (1974). L'acte tactique en jeu. Paris : Vigot

Mc Garry, T., Anderson, D., Wallace, S., Hugues, M \& Franks, I. (2002). Sport competition as a dynamical self-organizing system. Journal of Sports Sciences, 20, 771-781.

McMorris, T., Copeman, R., Corcoran, D., Saunders, G., \& Potter, S. (1993). Anticipation of soccer goalkeepers facing penalty kicks. In T. Reilly, J. Clarys, \& A. Stibbe (Eds), Science and Football II (pp. 250-253). London: E \& FN Spon.

Menaut, A. (1998). Le réel et le possible dans la pensée tactique. Talence : PUB.

Moles, A. (2002). Cybernétique et sciences sociales [CD-ROM]. Encyclopédie Universalis.

Mombaerts, E. (1991). De l'analyse du jeu à la formation du joueur de football. Joinville: ACTIO.

Mombaerts, E. (1999). Pédagogie du football Apprendre à jouer ensemble par la pratique du jeu. Paris: Vigot.

Mouchet, A. (2005). Modélisation de la complexité des décisions tactiques en rugby. eJRIEPS, 7, 3-19.

Palfai, J. (1989). Méthodes d'entraînement moderne en football. Bräkel : Broodcoorens.

Reboul-Marty, J., \& Launois, R. (1995). Les indicateurs de qualité de vie: Processus de mesure et validation. Cardioscopies, 33, 635-637.

Salembier \& Pavard, (2004) . Analyse et modélisation des activités coopératives situées. Evolution d'un questionnement et apports à la conception Lu le 30 mars 2005 dans : http://www.activites.org/v1n1/salembier.pdf. 
Savoyant, A., \& Bouthier, D. (1985). Contribution à la caractérisation de l'action collective sportive. In M. Laurent, \& P. Therme (Eds), Recherche en APS I (pp. 281-288). Marseille: Ed Centre de recherche UEREPS Aix Marseille II.

Shannon, C., \& Weaver, W. (1945). The mathematical theory of communication. Urbana : University of Illinois Press.

Vermersch, P. (2002). Quelques études de cas sur l'articulation entre situations d'étude et développement théoriques. Expliciter, 45, 1-11.

Walliser, B. (1977). Systèmes et Modèles. Introduction critique à l'analyse des systèmes. Paris : Le Seuil.

Wrzos, J. (1984). La tactique de l'attaque. Bräkel: Broodcoorens. 